\title{
Development of a novel Electro Active Polymer (EAP) actuator for driving the wings of flapping micro air vehicle
}

\author{
S. C. Burgess, C. S. Ling, A. Conn, S. Araromi, J. Wang \\ \& R. Vaidyanathan \\ Department of Mechanical Engineering, University of Bristol, UK
}

\begin{abstract}
This paper describes the development and testing of an EAP (electroactive polymer) actuator for driving the wings of an MAV (micro air vehicle). Creating the drive system for a flapping MAV is extremely challenging because of the required combined bending/twisting of the wing during flapping. Silicone-based DEs (dielectric elastomer) were used for the actuator material. In addition, the EAP actuators were operated at resonant frequency which is an important means of producing optimal flight performance with reduced power consumption. The paper presents the optimisation of the structural and operational performance of the EAP actuator. The paper also presents the test results of the actuator driving an actual MAV wing.
\end{abstract}

Keywords: electroactive polymer, micro air vehicle, resonant frequency.

\section{Introduction}

MAVs that use insect-like flapping for lift have the potential for achieving advanced flight capabilities such as silent operation; high payload; controlled hovering; and high manoeuvrability. Such flight performance is required for reconnaissance in confined places like buildings, urban areas, tunnels and caves. Insect-like flapping involves simultaneously flapping and twisting of the wings and the production of unsteady aerodynamic effects such as the leading edge vortex [1]. These unsteady aerodynamic effects are responsible for the high lift generated in insect flight. Creating a controllable MAV is extremely challenging because of the required combined bending/twisting wing movement. EAP 
actuators are well suited for MAVs because they are solid state actuators which have high strain outputs. They are an emerging technology and have the advantage that they are mechanically simple, compact and light-weight for many applications. In particular, they often do not require gearing in a drive system because of the adaptability of configuration and high strain output. Many existing flapping wing MAV designs are limited in performance because they use conventional constrained mechanisms like crank-rockers [2-5].

A key advantage of having a linear drive to produce the wing reciprocating motion is the ability to oscillate at resonant frequency. This can save energy by taking away the need to accelerate and decelerate the wings. The work by Goldfarb and colleagues at Vanderbilt University demonstrated the use of resonant flapping through the excitation of the torsional and bending natural frequencies using piezoelectric ceramic benders [6]. Similar resonant flapping demonstrators have also been reported in [7-9]. Another important advantage of linear actuators is that it is easier to adjust control surfaces such as the angle of attack of an individual wing as they are suited to driving under-constrained mechanisms.

\section{Optimisation of flapping wing drive system}

A schematic of a typical rotary drive actuation system such as a crank-rocker is shown in Fig. 1. Compliances and backlash in the drive system lead to shock loads and induced time lag from activation to output. Energy is also wasted on accelerating the masses of the various transmission components, effectively reducing efficiency.

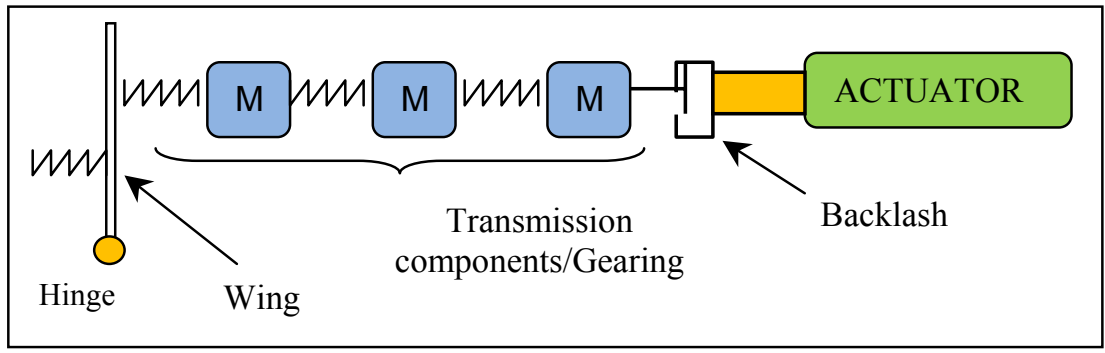

Figure 1: $\quad$ Typical rotary drive actuation system.

A schematic of the EAP linear actuation system developed at Bristol University is shown in Fig.2. The system is inherently more stiff and controllable.

An EAP or DE actuator is inherently well-suited for resonant operation because it is solid-state with no mechanisms and linkages. The DE bender designed in this project (Fig. 2) behaves like a cantilever beam (representing the shim substrate) with a uniform load (representing the elastomer film stack) acting across its length. The natural frequency for a cantilever beam uniform load can be calculated using equation (1): 


$$
f_{n}=\frac{K_{n}}{2 \pi} \sqrt{\frac{E I g}{w l^{4}}}
$$

where $w$ is the force per unit length of the uniform load (including shim substrate weight), $l$ is the bender free length, $E$ is the Young's modulus of the beam, $I$ is the moment of inertia, $g$ is the acceleration due to gravity and $K_{n}$ is 3.52 for the first mode of resonance.

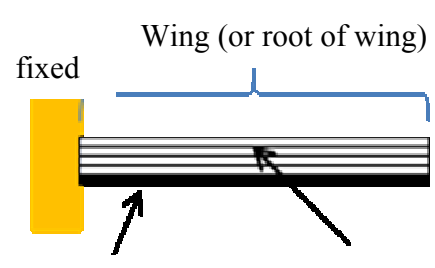

steel shim
DE layers



Active

Figure 2: $\quad$ EAP bender actuator developed at Bristol University.

For the DE bender configuration, the elastic modulus of the steel shim substrate is assumed to be $\mathrm{E}=200 \mathrm{GPa}$ and the inertia $\mathrm{I}=(\text { shim thickness })^{3} \times$ (shim width) $/ 12$, where the shim thickness $=50 \mu \mathrm{m}$ and shim width $=10 \mathrm{~mm}$. The bender free length, $l$, is $60 \mathrm{~mm}$ and the uniform load, $w$, is approximated as the actuator weight (equal to $39.2 \mathrm{~N}$ ) divided by $l$. Therefore, from the equation above, the natural frequency, $f_{\underline{n}}$, is approximately equal to $2.8 \mathrm{~Hz}$.

This closely matches the observed value during testing, which was approximately equal to $2.7 \mathrm{~Hz}$. Note that from equation (1) the actuator resonant frequency will increase with increase substrate moment of area and Young's modulus, however this would also decrease strain output. Therefore, there is a compromise between resonant frequency and strain output.

\section{Optimisation of actuator design for flapping}

\subsection{Material properties of DEs}

Most existing linear actuators are not suitable for MAVs because they require either additional strain amplification devices (e.g. piezo ceramic, conductive/electrorestrictive polymers) or have a limited power output (e.g. shape memory alloy, solenoid). However, DE actuators produce high strain at high power output. When an electric field is passed through the thin polymer film, sandwiched between two compliant electrodes, physical deformation takes place due to the effect of opposite charges attracting and like charges repelling. This causes the DE layer to be 'squeezed' in the transverse direction and expand in the planar direction. 
Silicone based Dow Corning HSIII and NuSil CF19-2186 have maximum volume specific energy densities of $160 \mathrm{~kJ} / \mathrm{m}^{3}$ and $220 \mathrm{~kJ} / \mathrm{m}^{3}$ respectively, and acrylic type elastomer 3M VHB 4910 has a maximum volume-specific energy of $3400 \mathrm{~kJ} / \mathrm{m}^{3}$ (estimated) [11]. For comparison, muscle has a maximum volumespecify energy of $70 \mathrm{~kJ} / \mathrm{m}^{3}$ [12]. Table 1 presents a comparison of properties of muscle and DEs. The table shows that EAPs have high stress and strain outputs.

Table 1: $\quad$ Comparison of performance of muscle and DEs [13].

\begin{tabular}{|l|l|l|}
\hline Property & Mammalian muscle & Dielectric elastomers \\
\hline Strain $(\%)$ & 20 & 10 to $>100$ \\
\hline Stress $(\mathrm{MPa})$ & $0.1-0.35$ & $0.1-2$ typical, max 3-9 \\
\hline Work density $\left(\mathrm{kJ} / \mathrm{m}^{3}\right)$ & $8-40$ & $10-150$ typical, max 3400 \\
\hline Density $\left(\mathrm{kg} / \mathrm{m}^{3}\right)$ & 1037 & $\sim 1000$ \\
\hline Strain rate $(\% / \mathrm{s})$ & $>50$ & $\begin{array}{l}450 \text { in } \mathrm{VHB}, 34000 \text { in } \\
\text { silicone }\end{array}$ \\
\hline Continuous power $(\mathrm{W})$ & $50-280$ & $\sim 500$ \\
\hline $\begin{array}{l}\text { Electromech. coupling } \\
(\%)\end{array}$ & $<40$ efficiency & 30 typical, max of 90 \\
\hline Cycle life & $>10^{9}$ & $10^{6} @, 50 \%$ strain \\
\hline Modulus $(\mathrm{MPa})$ & $10-60$ & $0.1-3$ \\
\hline Voltage $(\mathrm{V})$ & $<1 \mathrm{~V}$ & $>1 \mathrm{kV}$ \\
\hline
\end{tabular}

\subsection{Governing equations for DEs}

Using a simple electrostatic model, the pressure, $p$, exerted by the electrodes on the elastomer film is given in eqn. (2).

$$
p=e_{r} e_{0} T^{2}=e_{r} e_{0}(V / h)^{2}
$$

where $T$ is the applied electric field, $V$ is the applied voltage, $h$ is the elastomer film thickness, $e_{r}$ is the relative dielectric constant of the elastomer and $e_{0}$ is the permittivity of free space. From eqn. (2), the effective pressure, $p$, is essentially related to the force output of the $\mathrm{DE}$ actuator. For a given effective pressure, the higher the dielectric constant $\left(e_{r}\right)$ and the thinner the elastomer film is, the lower the electric field $(V)$ is needed to achieve this force. Assuming the elastomer film is unloaded and unconstrained, the thickness strain, $\varepsilon_{z}$, that results from $p$ is given by:

$$
\varepsilon_{z}=\frac{-p}{E}=\frac{-e_{r} e_{0}(V / h)^{2}}{E}
$$

where $\mathrm{E}$ is the Young's Modulus of the elastomer. 


\subsection{The bender DE actuator developed at Bristol University}

The bender produced in this work consists of a thin passive substrate (steel shim of $50 \mu \mathrm{m}$ in thickness) with layers of active DE with carbon electrode on the top surface. The electrode layers were stacked with alternating polarity, creating the active layers under activation. Matching polarity active layers were connected electrically using pre-cut aluminium strips, while self adhesive copper tape was used in the final stage to provide the electrical interface for the high voltage power supply. The composite beam bends when the EAP material is activated.

An in-house fabrication technique has been developed for production of thin silicone films. Two types of silicone DEs (BJB TC-5005, Dow Corning Silastic 3481 ) and one type acrylic DE (3M VHB 4910) have been acquired. The treatment and fabrication technique for each of these DEs are different. Films with thicknesses of 100,200 and $500 \mu \mathrm{m}$ have been produced by rolling the Kbar on the BJB TC-5005 silicone mixture. A similar technique, however, was found to be inappropriate for the Dow Corning Silastic 3481 (HSIII equivalent) which has higher surface tension. The mixture of the Silastic 3481 silicone (part A) and the curing agent (part B) has to be 'dissolved' in the solvent, and sprayed onto the steel substrate using an air brush. The 3M VHB 4910 acrylic elastomer, on the other hand, comes pre-fabricated with minimum thickness of $500 \mu \mathrm{m}$ and thus requires pre-straining biaxially before the compliant electrode can be applied.

\subsection{DE bender design considerations}

The DE actuator requires a high field strength, typically in the range of 50$200 \mathrm{MV} / \mathrm{m}$. The recent miniaturisation of step-up voltage converters (e.g. EMCO Q50-5) make them convenient and feasible for use in MAVs. The requirement for minimising the film thickness to reduce voltage requirements, coupled with the elastomer's finite breakdown voltage, means that a stacked configuration is necessary to achieve sufficient power output.

It is also essential to have highly compliant electrodes which maintain good conductivity under high strain rates. Several possible conductive materials have been identified for the electrode of the DE bender. These are grease doped with carbon or silver, silicone and carbon powder mix, Metal rubber ${ }^{\mathrm{TM}}$ (Nanosonic Inc., USA) and spluttered gold layers. The use of Metal rubber ${ }^{\mathrm{TM}}$ is limited by its high cost. Gold is difficult to apply because gaps have to be introduced during spluttering due to its high stiffness. Conductive grease is not suitable for stacking elastomers, as grease does not provide adhesion between the layers and that causes the layers to delaminate. A mixture of the silicone BJB TC-5005 with carbon was found to be the most appropriate candidate for the stack elastomer configuration.

The bender configuration is mass efficient (without hinges) and the passive substrate (steel shim) provides elastic storage. The multi-layered bender configuration pursued in this work benefits from the high power output of stacked films, combined with the high strain output and an elastic storage capability, make the choice of such configuration an optimal design. 


\section{DE bender and flapping wing configurations}

\subsection{Single degree of freedom flapping}

\section{Configuration 1}

The first and most basic configuration attempted to provide flapping using a single degree of freedom (DOF) multi-layered DE actuator with a scaled model wing attached rigidly to the actuator tip. The actuator produced ample force to flap the wing and overcome inertial forces. The actuation system was also able to be driven at resonant frequency and a maximum sweep angle of $40^{\circ}-50^{\circ}$ was observed in testing using an 8-layer actuator stack.

\section{Configuration 2}

To achieve strain amplification a simple lever mechanism was constructed. A carbon fibre rod was attached perpendicular to the protruding end of the leading edge wing spar. The rod was inserted into an aluminium tube with an inner diameter larger than that of the rod, therefore allowing it to rotate freely, thus facilitating the pivot for the lever mechanism as shown in Fig. 3.


Figure 3: $\quad$ EAP actuator with lever mechanism for strain amplification.

The prototype built was capable of being driven at resonance. Moreover, the moment produced by the mass of the wings provided some pre-tensioning on the $\mathrm{DE}$ actuator and the actuator motion worked with the natural rotation produced by the induced moment arm of the wing's mass. This resistance is primarily due to friction forces at the interface which may be overcome by rigidly attaching the wing spar to the actuator tip. However, the motion of the spar relative to the actuator is planar due to the curved profile of the activated actuator; hence the design of such a linkage is nontrivial.

\subsection{Flapping with passive twist}

The two configurations investigated above were adapted to allow for passive twisting of the wing about the length axis of the leading edge wing spar. The twisting is a result of aerodynamic forces and wing inertia. In insects, such as the Libellula pulchella dragonfly, these forces are sufficient to provide the wing pitching necessary for steady flight, without the aid of muscles [14].

\section{Configuration 3}

A design was constructed which allowed for twisting by placing the protruding end of the leading edge wing spar into the small length of aluminium tubing 
rigidly attached to the tip of the actuator. The mechanism is similar to that used to provide the pivot in Configuration 2, allowing for free rotation about the leading edge of the spar axis. A pitching angle of approximately $\pm 5^{\circ}$ was observed with this design in simple flapping test.
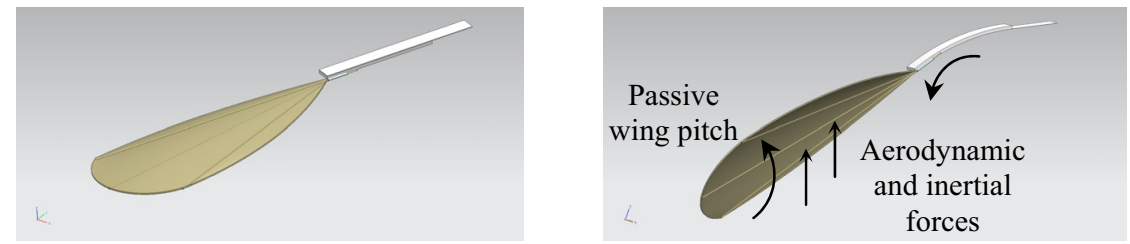

Figure 4: $\quad$ Wing flapping with passive twist.

\section{Configuration 4}

Configuration 3 can be adapted to incorporate the strain amplification mechanism utilised in Configuration 2, thus generating a greater sweep angle whilst maintaining passive pitching capabilities.

\subsection{Wing flapping with controlled twist}

Although there is evidence to show that passive wing pitching induced by aerodynamic and inertial forces may be sufficient for steady state flight applications, the ideal case would be to have dynamic control over wing pitching angle in addition to simple flapping motion.

\section{Configuration 5}

Configuration 5 was developed using a modified version of Configuration 3 with the addition of a second EAP actuator in parallel with the first. Initially, the two actuators were arranged so that they would act in opposite direction when activated, causing the wing to twist as a result.
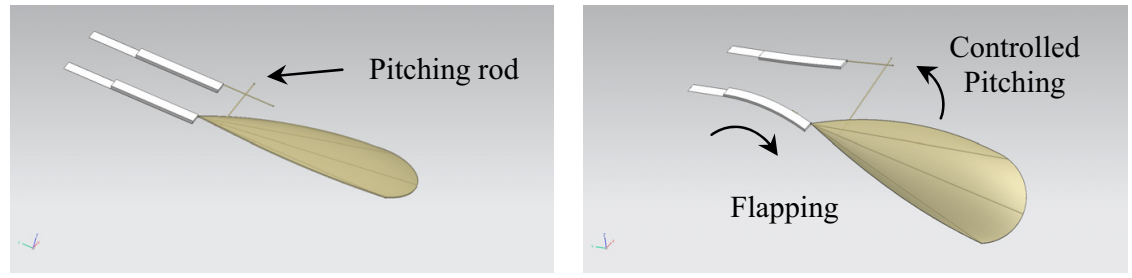

Figure 5: $\quad$ Flapping with controlled twist.

While this design is rather promising, the resistance to motion arising from frictional forces resulted in very little pitching being produced. The actuators also appeared to work against each other and produced no flapping. In an attempt to rectify this problem, the actuators were repositioned so that they acted in the same direction when activated, with one of two actuators given a slightly reduced voltage input to create a difference in sweep angle. In this case, friction in the system caused the actuators to behave as though they were rigidly attached via the pitching rod and therefore no twisting was produced. 


\subsection{Configuration summary}

Proof of concept flapping wing designs have been developed using DE benders. Configuration 3 shows good potential for further development with minimal design complexity. The limiting factor in the more complex designs was friction which in some cases, greatly reduced strain output. Moreover, future DE actuator designs will be investigated for two degree of freedom actuation. Such an actuator would also increase dynamic control of the wing motion whilst maintaining design simplicity.

\section{Experimental results}

To evaluate the performance of the DE bender, a simple strain test to measure the bender tip deflection was used. A number of stacked benders, comprising of 4, 8 and 12 layers of Dow Corning Silastic 3481 were fabricated. Each of these DE benders was placed inside a high-voltage isolation box with an EMCO F12TR high voltage converter. The benders were clamped on one end using a mounting block, while graph paper with $2 \mathrm{~mm}$ grid was placed directly under the bender. The supply voltage to the bender was incrementally increased from 0 $7.2 \mathrm{kV}$, and the tip deflection for each of these benders was captured using a digital camera mounted perpendicularly to the $2 \mathrm{~mm}$ grid. In between the measurements, the power supply was switched off to allow the bender to return to its original inactive position. This process was repeated for each bender and Fig. 6 shows the inactive and active state of the 12-layer bender.
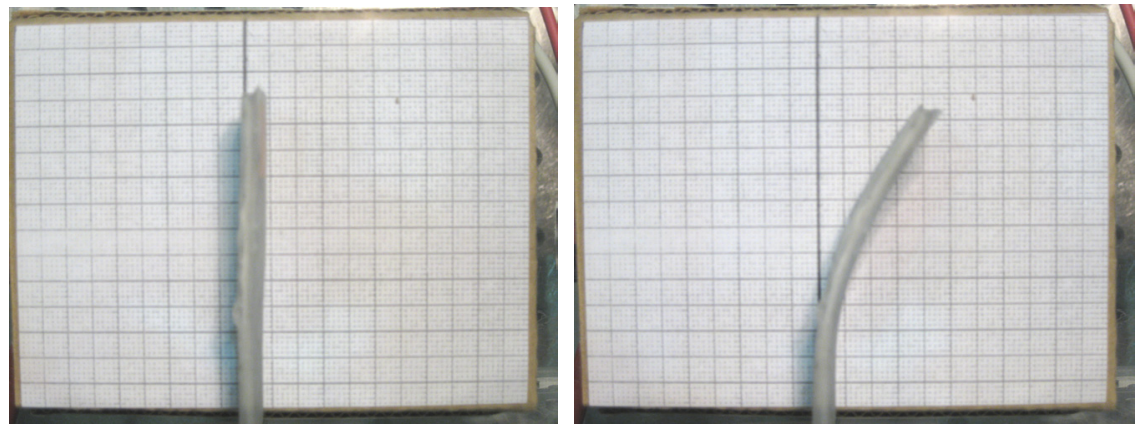

Figure 6: Dow Corning Silastic 3481 12-layer stacked bender when it is inactive (left). Tip displacement for the bender for a voltage of $6.4 \mathrm{kv}$ (right).

\subsection{Tip displacement measurements}

The tip deflection measurements for the 4 and 8 and 12-layer are shown in Fig. 7. The 4 and 8 and 12-layer bender produce maximum tip deflection of $2.4 \mathrm{~mm}$

$7.3 \mathrm{~mm}$ and $19.2 \mathrm{~mm}$ respectively. 


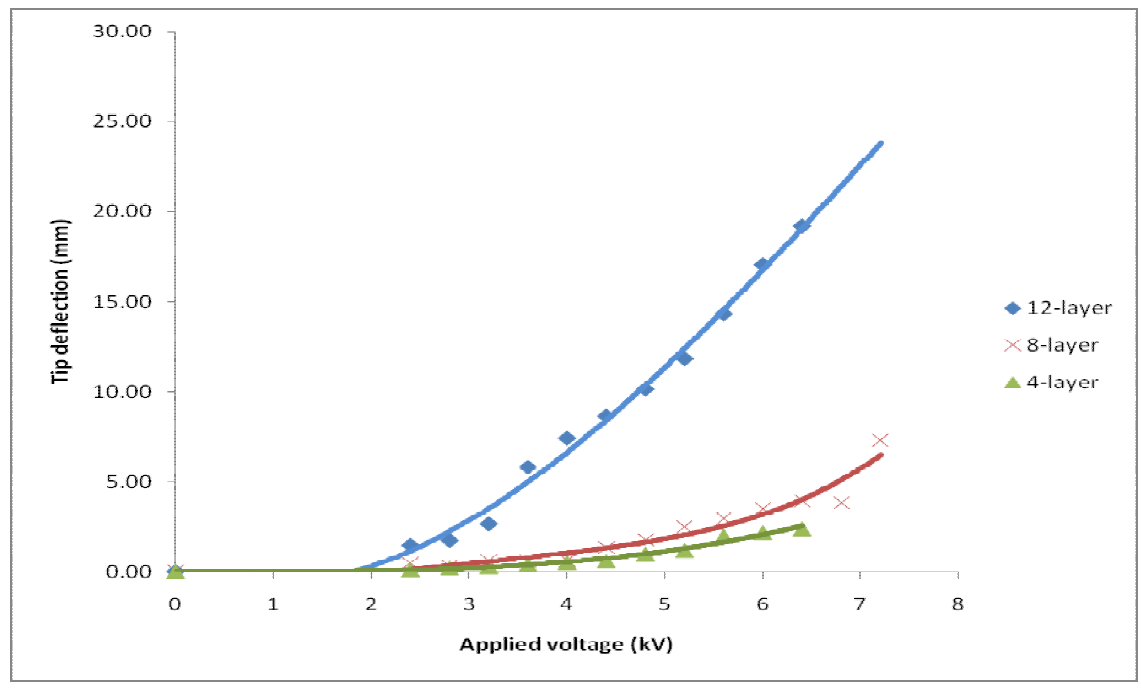

Figure 7: Tip displacement vs input voltage $(\mathrm{kV})$ for 4,8 and 12-layer DE bender.
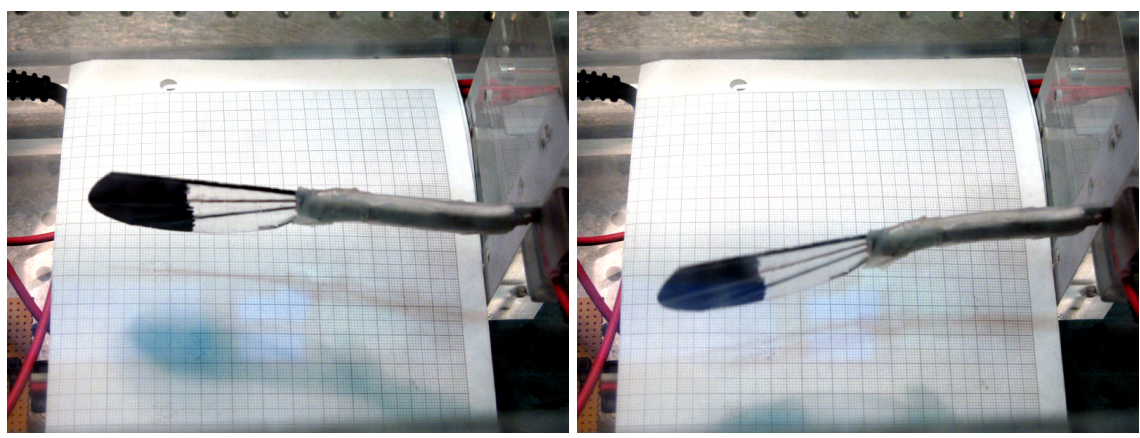

Figure 8: $\quad$ DE bender with wing attached during resonant operation.

\subsection{DE bender with wing in resonant operation}

A wing with spars made of thin carbon fibre rods and a Mylar membrane was attached to the end tip of the bender (same arrangement as Configuration 1 in Section 4.1). The actuator was driven by a square wave input with an adjustable frequency in an attempt to resonate the wing. A resonant frequency of approximately $3 \mathrm{~Hz}$ was identified for a 12-layer DE stacked bender (Fig. 8). When the voltage is turned off, the energy stored in the substrate (due to its stiffness) the bender-wing arrangement returns naturally to its original position. The momentum acquired during the upstroke cycle causes the wing to travel further than its original position, resulting in a reciprocating wing motion. When the actuator stack is oscillating at its natural frequency, the resonant operation has the effect of increasing stroke amplitude whilst minimising inertial energetic 
expenditure. The moment of inertia or stiffness of the bender will have to be increased in order to achieve the desired $20-30 \mathrm{~Hz}$ operation frequency for MAV.

\section{Conclusion}

The high volume-specific power density and high strain performance of DEs make them an inherently good choice of actuator for flapping wing MAVs. The ability to resonate EAPs as a solid state structure gives potential for high efficiency of operation. Resonance has been demonstrated at around $3 \mathrm{~Hz}$ for the bender actuator developed in this project.

Various configurations of bender actuators were designed, fabricated and tested. Configuration 3 was the simplest and allowed for flapping and passive wing pitching which is induced by aerodynamic and inertial forces. Preliminary test results show that the DE actuator exhibits potential for producing an optimally design flapping mechanism. Further improvements of the actuator configuration and fabrication techniques are needed to achieve actual flight of an MAV.

\section{References}

[1] Sane, S.P., The aerodynamics of insect flight. Journal of Experimental Biology, 206, pp. 4191-4208, 2003.

[2] Conn, A. T., Burgess, S. C., Ling, C. S. \& Vaidyanathan, R., The design optimisation of an insect- inspired micro air vehicle. Journal of Design \& Nature, WIT, 3(1), pp 12 - 27, 2008.

[3] Conn, A. T., Burgess, S. C., and Ling, C. S., Design of a parallel crankrocker flapping mechanism for insect-inspired micro air vehicles. Proc. IMechE, Part C: Journal of Mechanical Engineering Science, 221, Part C, pp. 1211-1222, 2007.

[4] Jones, K.D. and Platzer, M.F., Bio-inspired design of flapping wing micro air vehicles - an engineer's perspective. $44^{\text {th }}$ Aerospace Sciences Meeting and Exhibit, 9-12 Jan, Reno, 2006.

[5] Lentink, D., Bradshaw N.L. and Jongerius, S.R, Novel micro aircraft inspired by insect flight. Comparative Biochemistry and Physiology Part A, 146A, Supplement 1 (April 2007), pp. S133-S134, 2007.

[6] Cox, A., Monopoli, D., Cveticanin, D., Goldfarb, M., and Garcia, E., The development of elastodynamic components for piezoelectrically actuated flapping micro-air vehicles. Journal of Intelligent Material Systems and Structures, 13, pp. 611-615, 2002.

[7] Luc-Bouhali, A. Progress of the REMANTA project on MAV with flapping wings. European Micro Air Vehicle (EMAV) Conference and Flight Competition, Jul 25-26, Braunschweig, 2006.

[8] Syaifuddin, M., Park, H.C. and Goo, N.S., Design and evaluation of a LIPCA-actuated flapping device. Smart Materials and Structures, 15, pp. 1225-1230, 2006 
[9] Wood, R.J., Steltz, E. and Fearing, R.S., Optimal energy density piezoelectric bending actuators. Sensors and Actuators A: Physical, 119, pp. 476-488, 2005.

[10] Avadhanula, S., Wood, R.J., Steltz, E., Yan, J. and Fearing, R.S., Lift force improvements for the micromechanical flying insect. IEEE International Conference on Intelligent Robots and Systems, Oct 28-30, Las Vegas, 2003.

[11] Pelrine, R., Kornbluh, R., Joseph, J., Heydt, R., Pei, Q. and Chiba, S., Highfield deformation of elastomeric dielectrics for actuators. Materials Science and Engineering C, 11, pp. 89-100, 2000.

[12] Bar-Cohen, Y, Electroactive Polymer (EAP) Actuators as Artificial Muscle, 2nd edn. SPIE, Bellingham, WA, 2004.

[13] Pei, Q., Rosenthal, M. A., Pelrine, R., Stanford, S. and Kornbluh, R. D., Multifunctional electroelastomer roll actuators and their application for biomimetic walking robots [5051-31]. Proceeding of SPIE, 5051, pp. 281290, 2003.

[14] Bergou A.J., Xu, S., Wang, Z. J. (2007). Passive wing pitch reversal in insect flight. Journal of Fluid dynamics, 59, pp. 321-337, 2007. 